\title{
A variational analysis for large deflection of skew plates under uniformly distributed load through domain mapping technique
}

\author{
Debabrata Das, Prasanta Sahoo* and Kashinath Saha
}

Department of Mechanical Engineering, Jadavpur University, Kolkata - 700032, India.

Corresponding author (e-mail: psahoo@vsnl.net,psjume@gmail.com, Tele-fax: +913324146890)

\begin{abstract}
In the present paper, the static behaviour of thin isotropic skew plates under uniformly distributed load is analyzed with the geometric nonlinearity of the model properly handled. A variational method based on total potential energy has been implemented through assumed displacement field. The computational work has been carried out on a square normalized domain based on an appropriate domain mapping technique. Validation study for the present work has been carried out quite extensively to establish its accuracy and stability. The developed method is quite general to be applied readily for any classical boundary condition, but to maintain brevity results have been furnished for clamped and simply supported boundaries only.
\end{abstract}

Keywords: Skew plate; Variational method; Domain mapping; Large deflection; Jacobian

\section{Introduction}

Skew plates have quite a good number of applications in modern structures. Skew plate structures can be found frequently in modern construction in the form of reinforced slabs or stiffened plates. Such structures are widely used as floors in bridges, ship hulls, buildings, etc. Skew plates are also used in the construction of wings, tails and fins of aircrafts and missiles. Simulation of static behaviour of skew plates is an interesting area of work for the researchers.

Alwar and Rao (1973) presented the non-linear analysis of orthotropic skew plates of constant thickness subjected to uniform transverse load. They developed a numerical technique of dynamic relaxation to carry out the analysis. Dynamic relaxation is essentially a step by step integration of critically damped vibration using viscous damping to ensure the attainment of a steadystate solution. Alwar and Rao (1974) used the same technique of dynamic relaxation. They have investigated the non-linear behaviour of clamped isotropic skew plate of constant thickness under uniformly distributed load. Srinivasan and Ramachandran (1976) presented large deflection elastic behaviour of clamped, uniformly loaded orthotropic skew plates. They obtained the solution by using Newton-Raphson method to solve the non-linear algebraic equations transformed from governing non-linear partial differential equations. Buragohain and Patodi (1978) investigated the large deflection skew plate problem and formulated it by energy principle to derive a set of nonlinear algebraic equations which are solved by using Newton-Raphson iterative method with incremental loading. They claimed their formulation to be independent of boundary conditions and furnished results for clamped and simply supported isotropic skew plates of constant thickness with immovable edges and subjected to uniformly distributed transverse load. Chia (1980) considered the moderately large deflection elastic behaviour of homogenous isotropic and laminated anisotropic rectangular as well as skew plates by analytical method. Xiang et al. (1995) studied the elastic buckling behaviour of skew Mindlin plate under shear load. Based on the Rayleigh-Ritz method, new critical shear load factors and buckling mode shapes for two kinds of shear loads - rectangular shear (R-shear) and skew shear (S-shear) - have been investigated.

Liew and Han (1997) presented the bending analysis of a simply supported thick skew plate based on the first-order shear deformation using Reissner/Mindlin theory. They introduced the geometric transformation of the governing differential equations and boundary conditions of the plate from the physical domain into a unit square computational domain. Subsequently they derived a set of linear algebraic equations from the transformed differential equations via differential quadrature method (DQM) and the approximate solutions of the problem are obtained by solving the set of algebraic equations. Using Boundary Element Method 
(BEM), a fundamental solution in oblique coordinates for the analysis of isotropic skew plates under transverse loading has been obtained by Rajamohan and Raamachandran (1997) based on the analogy with the anisotropic plates bending governing equation in Cartesian coordinates. They have used Charge Simulation Method (CSM), a version of BEM, to obtain the solution and the simplicity of the method was demonstrated by avoiding numerical integration due to the fact that the domain integrals, which appear in any boundary element method is avoided by assuming a suitable polynomial particular integral function.

Saadatpour et al. (1998) developed a numerical method for the stability analysis of arbitrary quadrilateral-shaped elastic plates with internal supports and used the Galerkin method in conjunction with the natural coordinates of the plate as a basis for the analysis of general plates. Wang et al. (2003) proposed a new version of differential quadrature method (DQM) to obtain buckling loads of thin anisotropic rectangular and isotropic skew plates for clamped and simply supported boundary conditions. In this new technique, two degrees of freedom in boundary points for determination of the weighting coefficients have been introduced in the existing differential quadrature element method (DQEM). Duan and Mahendran (2003) had developed a new non-linear quadrilateral hybrid/mixed shell element with five degrees of freedom at each node, using oblique coordinate systems to analyze the large deflection behavior of skew plate under uniformly distributed and concentrated loads. The authors have claimed to overcome the shortcomings for carrying out the large deflection analyses of the skew plates that have a $60^{\circ}$ skew angle under a concentrated load. Singh and Elaghabash (2003) presented a numerical method for the linear and geometrically nonlinear static analysis of thin plates having quadrangular boundary with four straight edges. They have derived the matrix equation of equilibrium using the work-energy principle with the assumed displacement fields expressed by algebraic polynomials.

Liew et al. (2004) employed a mesh-free radial basis function (RBF) method for the buckling analysis of non-uniformly loaded thick plates. They obtained initial (i.e., pre-buckling) stresses by discretizing the variational form of the static system of equations and calculated the static buckling loads of the plates by solving the resultant Eigen value equation. Muhammad and Singh (2004) presented an energy method for the linear static analysis of first order shear deformable plates of various shapes. In this method, the displacement fields are defined in terms of the shape functions, which correspond to a set of predefined points and are composed of significantly higher order polynomials. Malekzadeh and Fiouz (2007) have presented two different differential quadrature (DQ) approaches based on the thin plate theory (TPT-DQ) and the first order shear deformation plate theory (FSDTDQ) to investigate the large deformation analyses of thin and moderately thick orthotropic skew plates with rotationally restrained edges. In both approaches, they have modeled the geometrical nonlinearity of the plate by using Green's strain and Von Karman assumption. Daripa and Singha (2009) studied the influence of corner stresses on the stability behaviour of composite skew plates. Prakash et al. (2008) analyzed thermal post-buckling of skew plates having functionally graded materials while Wu and Shih (2006) considered the dynamic instability of arbitrarily laminated skew plates.

Several researchers have addressed the linear and non-linear dynamic problems of skew plates incorporating various complicating effects. Singh et al. (2006) considered the vibration behaviour of thermally stressed composite plates whereas Ashour (2009) analyzed free vibration behaviour of symmetrically laminated clamped skew plates. Das et al. (2008, 2009) used a variational method to study large amplitude dynamic problem of skew plates under simply supported and clamped boundary conditions. Differential quadrature method has also been used for non-linear vibration problems of laminated composite skew plates (Malekzadeh, 2007, 2008; Malekzadeh and Karami, 2006).

In the present work, a simulation model for large deflection static analysis of a thin isotropic skew plate under uniform transverse pressure has been presented. The mathematical formulation is based on variational form of energy principle. The displacement fields are assumed as orthogonal polynomials in the computational domain that satisfy the plate boundary conditions and the resulting nonlinear set of equations are solved by direct substitution method using relaxation parameter. Results have been validated successfully with the available ones and a good amount of new results has been presented which will be of great help to designers. It is found that convergence in results for large skew angle plates poses numerical difficulty and to this end a new extrapolation technique has been prescribed.

\section{Mathematical formulation}

A skew plate $(a \times b \times t)$ with skew angle $\theta$ is shown in Figure 1. For large displacement formulation, it is assumed that the material of the plate is isotropic and homogeneous. The thickness of the plate is considered to be sufficiently small so as to avoid the effect of shear deformation. Also the stress and strain measures are based on the original dimensions of the plate.

\subsection{Mapping of physical domain into computational domain}

The physical domain of interest is mapped to the computational domain in $\xi-\eta$ coordinate system as shown in Figure 2. In this domain, the intersections of constant $\xi$ and constant $\eta$ lines passing through the gauss points are selected as the reference points for computation. The coordinates of all the gauss points in the computational domain are calculated numerically in the $x-y$ plane by suitable grid generation techniques. This technique of numerical grid generation is not used extensively by any other researcher, and it can be used for similar other problems involving irregular geometry such as rectangular plate with a central circular cut-out. 


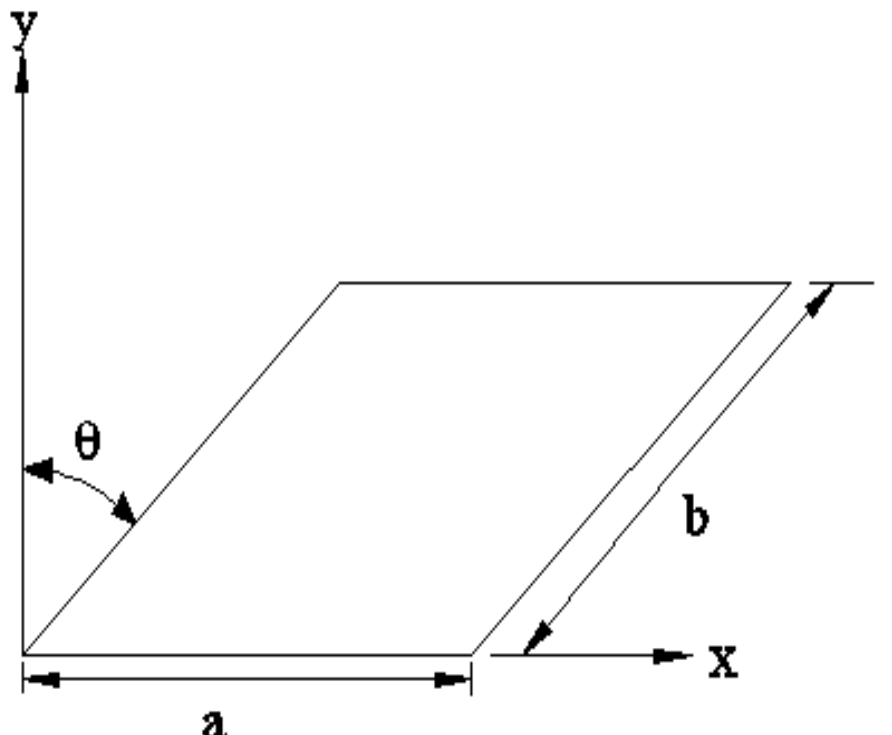

Figure 1. Skew plate in $x-y$ plane.

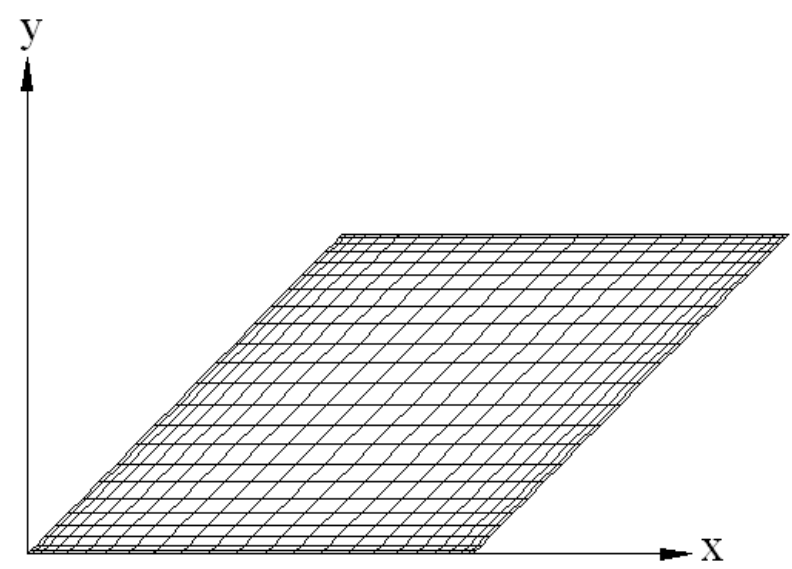

(a) Physical domain

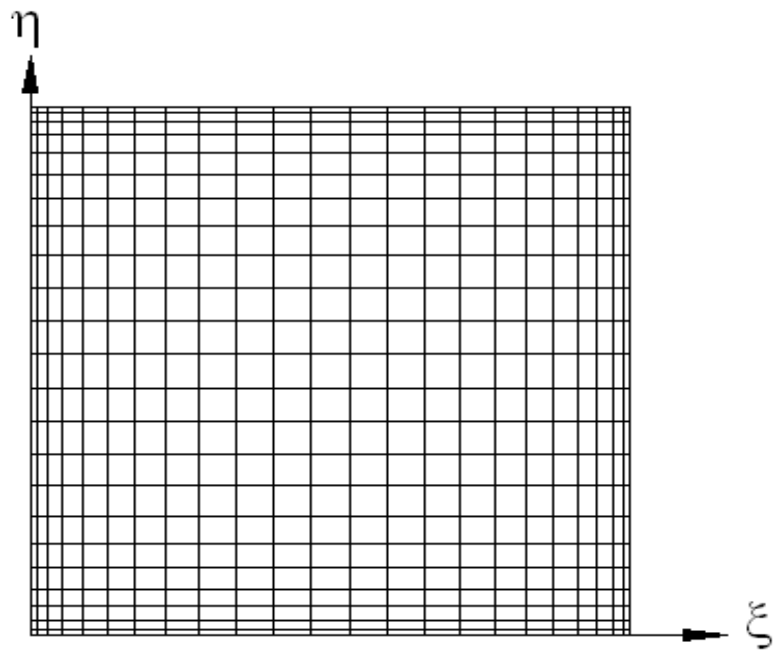

(b) Computational domain

Figure 2. Gauss point locations for skew plate. 


\subsection{Variational principle}

Variational principle states that

$$
\delta(U+V)=0
$$

where, $U=U_{b}+U_{m}$ is total strain energy, $U_{b}$ is strain energy due to pure bending and $U_{m}$ is strain energy due to stretching of its middle surface, $V$ is the potential energy of the external forces and $\delta$ is the variational operator in configuration space. It is to be noted here that geometric nonlinearity, i.e., the nonlinear strain-displacement relationship is taken into consideration through inplane stretching.

$U_{b}$ is given by (Timoshenko and Woinowsky, 1959),

$U_{b}=\frac{D^{2}}{2} \int_{0}^{b \cos \theta} \int_{y \tan \theta}^{y \tan \theta+a}\left[\left\{\left(\frac{\partial^{2} w}{\partial x^{2}}\right)^{2}+\left(\frac{\partial^{2} w}{\partial y^{2}}\right)^{2}+2 \frac{\partial^{2} w}{\partial x^{2}} \frac{\partial^{2} w}{\partial y^{2}}\right\}-2(1-v)\left\{\frac{\partial^{2} w}{\partial x^{2}} \frac{\partial^{2} w}{\partial y^{2}}-\left(\frac{\partial^{2} w}{\partial x \partial y}\right)^{2}\right\}\right] d x d y$

where, $D=\frac{E t^{3}}{12\left(1-v^{2}\right)}$, flexural rigidity of plate and $u, v$ and $w$ are components of displacement along $\mathrm{x}, \mathrm{y}$ and $\mathrm{z}$ directions respectively.

$U_{m}$ is given by (Timoshenko and Woinowsky, 1959),

$U_{m}=\frac{E t}{2\left(1-v^{2}\right)} \int_{0}^{b \cos \theta} \int_{y \tan \theta}^{\tan \theta+a}\left[\left\{\left(\frac{\partial u}{\partial x}\right)^{2}+\left(\frac{\partial v}{\partial y}\right)^{2}+\frac{\partial u}{\partial x}\left(\frac{\partial w}{\partial x}\right)^{2}+\frac{\partial v}{\partial y}\left(\frac{\partial w}{\partial y}\right)^{2}\right\}+\frac{1}{4}\left\{\left(\frac{\partial w}{\partial x}\right)^{2}+\left(\frac{\partial w}{\partial y}\right)^{2}\right\}\right.$
$\left.+2 v\left\{\frac{\partial u}{\partial x} \frac{\partial v}{\partial y}+\frac{1}{2} \frac{\partial v}{\partial y}\left(\frac{\partial w}{\partial x}\right)^{2}+\frac{1}{2} \frac{\partial u}{\partial x}\left(\frac{\partial w}{\partial y}\right)^{2}\right\}+\frac{1-v}{2}\left\{\left(\frac{\partial u}{\partial y}\right)^{2}+\left(\frac{\partial v}{\partial x}\right)^{2}+2 \frac{\partial u}{\partial y} \frac{\partial v}{\partial x}+2\left(\frac{\partial u}{\partial y}+\frac{\partial v}{\partial x}\right) \frac{\partial w}{\partial x} \frac{\partial w}{\partial y}\right\}\right] d x d y$

For a plate loaded with uniform transverse pressure $p$, potential energy $V$ is given by,

$$
V=-\int_{0}^{b \cos \theta} \int_{y \tan \theta}^{y \tan \theta+a}(p w) d x d y
$$

\subsection{Approximate displacement field}

The displacement fields $w, u$ and $v$ are expressed by linear combinations of unknown parameters $d_{i}$ as follows:

$$
\begin{aligned}
& w(\xi, \eta)=\sum_{i=1}^{n w} d_{i} \phi_{i}(\xi, \eta), \\
& u(\xi, \eta)=\sum_{i=n w+1}^{n w+n u} d_{i} \alpha_{i-n w}(\xi, \eta), \\
& v(\xi, \eta)=\sum_{i=n w+n u+1}^{n w+n u+n v} d_{i} \beta_{i-n w-n u}(\xi, \eta)
\end{aligned}
$$

where, $\phi(\xi, \eta), \alpha(\xi, \eta)$ and $\beta(\xi, \eta)$ are sets of orthogonal functions and $n w, n u$ and $n v$ are number of functions for $w, u$ and $v$ respectively.

The start functions of these orthogonal sets are selected to satisfy the flexural and membrane boundary conditions of the plate. As the entire computation is carried out in normalized computational domain, all the start functions are defined in computational domain. The basic functions for the definition of plate deflection $w$ come from the flexural boundary conditions, either clamped or simply supported. The start functions for $u$ and $v$ are selected to satisfy the zero displacement conditions at the boundary, being considered as the only form of in-plane boundary condition. The higher order functions are generated following a two dimensional implementation of Gram-Schmidt scheme and this is done for the first time in this type of study.

In conventional method of generating higher order functions, first the higher order one dimensional functions are generated from the corresponding start functions and then the higher order two dimensional functions are generated by ordered multiplication of the one dimensional functions corresponding to two orthogonal directions. In the present method, higher order functions in a particular orthogonal direction are generated corresponding to different coordinate values in the other orthogonal direction through Gram-Schmidt scheme. Then these values at a particular coordinate are collected to generate the complete set of higher order twodimensional functions through the implementation of Gram-Schmidt scheme. The advantage of using this technique is that unlike the earlier case, the start functions can be of two variables that need not be separable in spatial coordinates. 


\subsection{Derivation of governing system of equations}

The governing equations are obtained in the following form using Eqs. (2), (3), (4) and (5) in Eq. (1),

$$
[K]\{d\}=\{f\}
$$

where, $[K]=\left[k^{b}\right]+\left[k^{m}\right]$ is the stiffness matrix of dimension $(n w+n u+n v),\left[k^{b}\right]$ is the stiffness matrix due to bending action, $\left[k^{m}\right]$ is the stiffness matrix due to stretching action, $\{d\}$ is the unknown coefficient vector of dimension $(n w+n u+n v)$ and $\{f\}$ is the load vector of dimension $(n w+n u+n v)$.

$\left[k^{b}\right]$ is of the form given below:

$$
\left[k^{b}\right]=\left[\begin{array}{lll}
k_{11}^{b} & k_{12}^{b} & k_{13}^{b} \\
k_{21}^{b} & k_{22}^{b} & k_{23}^{b} \\
k_{31}^{b} & k_{32}^{b} & k_{33}^{b}
\end{array}\right]
$$

where,

$$
\begin{aligned}
{\left[k_{11}^{b}\right]=} & D \sum_{j=1}^{n w} \sum_{i=1}^{n w} \int_{0}^{1} \int_{0}^{1}\left[\left\{\left(\frac{\partial^{2} \phi_{i}}{\partial x^{2}}\right)\left(\frac{\partial^{2} \phi_{j}}{\partial x^{2}}\right)+\left(\frac{\partial^{2} \phi_{i}}{\partial y^{2}}\right)\left(\frac{\partial^{2} \phi_{j}}{\partial y^{2}}\right)+\left(\frac{\partial^{2} \phi_{i}}{\partial y^{2}}\right)\left(\frac{\partial^{2} \phi_{j}}{\partial x^{2}}\right)\right.\right. \\
& \left.+\left(\frac{\partial^{2} \phi_{i}}{\partial x^{2}}\right)\left(\frac{\partial^{2} \phi_{j}}{\partial y^{2}}\right)\right\}-(1-v)\left\{\left(\frac{\partial^{2} \phi_{i}}{\partial y^{2}}\right)\left(\frac{\partial^{2} \phi_{j}}{\partial x^{2}}\right)+\left(\frac{\partial^{2} \phi_{i}}{\partial x^{2}}\right)\left(\frac{\partial^{2} \phi_{j}}{\partial y^{2}}\right)\right. \\
& \left.\left.-2\left(\frac{\partial^{2} \phi_{i}}{\partial x \partial y}\right)\left(\frac{\partial^{2} \phi_{j}}{\partial x \partial y}\right)\right\}\right] \operatorname{det} J d \xi d \eta \\
{\left[k_{12}^{b}\right]=} & {\left[k_{13}^{b}\right]=\left[k_{21}^{b}\right]=\left[k_{22}^{b}\right]=\left[k_{23}^{b}\right]=\left[k_{31}^{b}\right]=\left[k_{32}^{b}\right]=\left[k_{33}^{b}\right]=0 }
\end{aligned}
$$

$\left[k^{m}\right]$ is of the form given below:

$$
\left[k^{m}\right]=\left[\begin{array}{lll}
k_{11}^{m} & k_{12}^{m} & k_{13}^{m} \\
k_{21}^{m} & k_{22}^{m} & k_{23}^{m} \\
k_{31}^{m} & k_{32}^{m} & k_{33}^{m}
\end{array}\right]
$$

where,

$$
\begin{aligned}
{\left[k_{11}^{m}\right]=} & \frac{E t}{2\left(1-v^{2}\right)} \sum_{j=1}^{n w} \sum_{i=1}^{n w} \int_{0}^{1} \int_{0}^{1}\left[\left(\sum_{i=1}^{n w} d_{i} \frac{\partial \phi_{i}}{\partial x}\right)^{2} \frac{\partial \phi_{i}}{\partial x} \frac{\partial \phi_{j}}{\partial x}+\left(\sum_{i=1}^{n w} d_{i} \frac{\partial \phi_{i}}{\partial y}\right)^{2} \frac{\partial \phi_{i}}{\partial y} \frac{\partial \phi_{j}}{\partial y}\right. \\
& +\left(\sum_{i=1}^{n w} d_{i} \frac{\partial \phi_{i}}{\partial x}\right)\left(\sum_{i=1}^{n w} d_{i} \frac{\partial \phi_{i}}{\partial y}\right)\left\{\frac{\partial \phi_{i}}{\partial x} \frac{\partial \phi_{j}}{\partial y}+\frac{\partial \phi_{i}}{\partial y} \frac{\partial \phi_{j}}{\partial x}\right\}+(1-v)\left(\sum_{i=n w+1}^{n w+n u} d_{i} \frac{\partial \alpha_{i-n w}}{\partial y}\right) \frac{\partial \phi_{i}}{\partial x} \frac{\partial \phi_{j}}{\partial y} \\
& \left.+(1-v)\left(\sum_{i=n w+n u+1}^{n w+n u+n v} d_{i} \frac{\partial \beta_{i-n w-n u}}{\partial x}\right) \frac{\partial \phi_{i}}{\partial x} \frac{\partial \phi_{j}}{\partial y}\right] \operatorname{det} J d \xi d \eta \\
{\left[k_{12}^{m}\right]=} & \frac{E t}{2\left(1-v^{2}\right)} \sum_{j=1}^{n w} \sum_{i=n w+1}^{n w+n u} \int_{0}^{1} \int_{0}^{1}\left[2\left(\sum_{i=1}^{n w} d_{i} \frac{\partial \phi_{i}}{\partial x}\right) \frac{\partial \alpha_{i-n w}}{\partial x} \frac{\partial \phi_{j}}{\partial x}+2 v\left(\sum_{i=1}^{n w} d_{i} \frac{\partial \phi_{i}}{\partial y}\right) \frac{\partial \alpha_{i-n w}}{\partial x} \frac{\partial \phi_{j}}{\partial y}\right. \\
& \left.+(1-v)\left(\sum_{i=1}^{n w} d_{i} \frac{\partial \phi_{i}}{\partial y}\right) \frac{\partial \alpha_{i-n w}}{\partial y} \frac{\partial \phi_{j}}{\partial x}\right] \operatorname{det} J d \xi d \eta \\
{\left[k_{13}^{m}\right]=} & \frac{E t}{2\left(1-v^{2}\right)} \sum_{j=1}^{n w} \sum_{i=n w+n u+1}^{n w+n u+n v} \int_{0}^{1} \int_{0}^{1}\left[2\left(\sum_{i=1}^{n w} d_{i} \frac{\partial \phi_{i}}{\partial y}\right) \frac{\partial \beta_{i-n w-n u}}{\partial y} \frac{\partial \phi_{j}}{\partial y}+2 v\left(\sum_{i=1}^{n w} d_{i} \frac{\partial \phi_{i}}{\partial x}\right) \frac{\partial \beta_{i-n w-n u}}{\partial y} \frac{\partial \phi_{j}}{\partial x}\right.
\end{aligned}
$$




$$
\begin{aligned}
& \left.+(1-v)\left(\sum_{i=1}^{n w} d_{i} \frac{\partial \phi_{i}}{\partial y}\right) \frac{\partial \beta_{i-n w-n u}}{\partial x} \frac{\partial \phi_{j}}{\partial x}\right] \operatorname{det} J d \xi d \eta \\
& {\left[k_{21}^{m}\right]=\frac{E t}{2\left(1-v^{2}\right)} \sum_{j=n w+1}^{n w+n u} \sum_{i=1}^{n w} \int_{0}^{1} \int_{0}^{1}\left[2\left(\sum_{i=1}^{n w} d_{i} \frac{\partial \phi_{i}}{\partial x}\right) \frac{\partial \phi_{i}}{\partial x} \frac{\partial \alpha_{j-n w}}{\partial x}+v\left(\sum_{i=1}^{n w} d_{i} \frac{\partial \phi_{i}}{\partial y}\right) \frac{\partial \phi_{i}}{\partial y} \frac{\partial \alpha_{j-n w}}{\partial x}\right.} \\
& \left.+(1-v)\left(\sum_{i=1}^{n w} d_{i} \frac{\partial \phi_{i}}{\partial x}\right) \frac{\partial \phi_{i}}{\partial y} \frac{\partial \alpha_{j-n w}}{\partial y}\right] \operatorname{det} J d \xi d \eta \\
& {\left[k_{22}^{m}\right]=\frac{E t}{2\left(1-v^{2}\right)} \sum_{j=n w+1}^{n w+n u} \sum_{i=n w+1}^{n w+n u} \int_{0}^{1} \int_{0}^{1}\left[2 \frac{\partial \alpha_{i-n w}}{\partial x} \frac{\partial \alpha_{j-n w}}{\partial x}+(1-v) \frac{\partial \alpha_{i-n w}}{\partial y} \frac{\partial \alpha_{j-n w}}{\partial y}\right] \operatorname{det} J d \xi d \eta} \\
& {\left[k_{23}^{m}\right]=\frac{E t}{2\left(1-v^{2}\right)} \sum_{j=n w+1}^{n w+n u} \sum_{i=n w+n u+1}^{n w+n u+n v} \int_{0}^{1} \int_{0}^{1}\left[2 v \frac{\partial \beta_{i-n w-n u}}{\partial y} \frac{\partial \alpha_{j-n w}}{\partial x}+(1-v) \frac{\partial \beta_{i-n w-n u}}{\partial x} \frac{\partial \alpha_{j-n w}}{\partial y}\right] \operatorname{det} J d \xi d \eta} \\
& {\left[k_{31}^{m}\right]=\frac{E t}{2\left(1-v^{2}\right)} \sum_{j=n w+n u+1}^{n w+n u+n v} \sum_{i=1}^{n w} \int_{0}^{1} \int_{0}^{1}\left[2\left(\sum_{i=1}^{n w} d_{i} \frac{\partial \phi_{i}}{\partial y}\right) \frac{\partial \phi_{i}}{\partial y} \frac{\partial \beta_{j-n w-n u}}{\partial y}+v\left(\sum_{i=1}^{n w} d_{i} \frac{\partial \phi_{i}}{\partial x}\right) \frac{\partial \phi_{i}}{\partial x} \frac{\partial \beta_{j-n w-n u}}{\partial y}\right.} \\
& \left.+(1-v)\left(\sum_{i=1}^{n w} d_{i} \frac{\partial \phi_{i}}{\partial x}\right) \frac{\partial \phi_{i}}{\partial y} \frac{\partial \beta_{j-n w-n u}}{\partial x}\right] \operatorname{det} J d \xi d \eta \\
& {\left[k_{32}^{m}\right]=\frac{E t}{2\left(1-v^{2}\right)} \sum_{j=n w+n u+1}^{n w+n u+n v} \sum_{i=n w+1}^{n w+n u} \int_{0}^{1} \int_{0}^{1}\left[2 v \frac{\partial \alpha_{i-n w}}{\partial x} \frac{\partial \beta_{j-n w-n u}}{\partial y}+(1-v) \frac{\partial \alpha_{i-n w}}{\partial y} \frac{\partial \beta_{j-n w-n u}}{\partial x}\right] \operatorname{det} J d \xi d \eta} \\
& {\left[k_{33}^{m}\right]=\frac{E t}{2\left(1-v^{2}\right)} \sum_{j=n w+n u+1}^{n w+n u+n v} \sum_{i=n w+n u+1}^{n w+n u+n v} \int_{0}^{1} \int_{0}^{1}\left[2 \frac{\partial \beta_{j-n w-n u}}{\partial y} \frac{\partial \beta_{j-n w-n u}}{\partial y}+(1-v) \frac{\partial \beta_{j-n w-n u}}{\partial x} \frac{\partial \beta_{j-n w-n u}}{\partial x}\right] \operatorname{det} J d \xi d \eta}
\end{aligned}
$$

The load vector $\{f\}$ is of the form $\left[f_{11} f_{12} f_{13}\right]^{T}$, where,

$$
\left\{f_{11}\right\}=p \sum_{j=1}^{n w} \int_{0}^{1} \int_{0}^{1} \phi_{j} \operatorname{det} J d \xi d \eta
$$

and

$$
\left\{f_{12}\right\}=\left\{f_{13}\right\}=0 \text {, as there is no in-plane loading. }
$$

Here, $J$ is the Jacobian of the transformation from $x-y$ plane to $\xi-\eta$ plane given by,

$$
J=\left[\begin{array}{ll}
\left(\frac{\partial x}{\partial \xi}\right) & \left(\frac{\partial y}{\partial \xi}\right) \\
\left(\frac{\partial x}{\partial \eta}\right) & \left(\frac{\partial y}{\partial \eta}\right)
\end{array}\right]
$$

\section{Solution Methodology}

The set of governing equations (Eq. (6)) is nonlinear in nature and solved by direct substitution technique using successive relaxation scheme (Cook et al., 1989). For each load-step, the values of $\left\{d_{i}\right\}$ are assumed to evaluate the stiffness matrix. Using the stiffness matrix and based on assumed values, new values of $\left\{d_{i}\right\}$ are calculated by matrix inversion technique from the expression $\{d\}=[K]^{-1}\{f\}$. Calculated values of $\left\{d_{i}\right\}$ are compared with their values in the previous iteration. If the difference comes below a predefined value of error limit, $\mathcal{E}$, the convergence of deflection field is assumed, otherwise the values of $\left\{d_{i}\right\}$ are modified with a relaxation parameter and it is taken as next approximation for the values of $\left\{d_{i}\right\}$. Once the solution is obtained for a load, stress and strain fields are computed following routine procedure. Subsequently an increment is given on load and iteration starts with the present solution or with another assumed deflection field, which may be extrapolated from the previous 
solution set. In the present extrapolation technique, the ratios of the unknown coefficients $\left\{d_{i}\right\}$ for the preceding two load steps are used to calculate the guess values of $\left\{d_{i}\right\}$ at the present load step. Although this technique has not been tried extensively, this can be utilized as a useful numerical tool for the solution of nonlinear set of equations, when convergence gets difficult. The entire program for the present study is developed in Fortran.

\section{Results and Discussions}

All the results in this paper have been presented for uniform transverse pressure. Although, the present method is quite general to be applied for any classical boundary condition, normalized load-deflection plots are presented for skew plates for two types of flexural boundary conditions: all edges clamped (CCCC) and all edges simply supported (SSSS). In both the cases, the edges of the plate are modeled as immovable by imposing zero in-plane displacement boundary conditions. Here ' $C$ ' and ' $S$ ' denote clamped and simply supported boundaries, respectively, for a single edge of the plate. Results for different possible combinations of clamped and simply supported boundary conditions are omitted here to maintain brevity, but it will be taken up in the subsequent study. The normalized load $\left(p^{*}\right)$ is defined as $p^{*}=\left(p a^{4}\right) /(16 D t)$ and the maximum normalized deflection is defined as $w^{*}=w_{\max } / t$. The results are generated using the following material and geometric parameter values, $E=210 \mathrm{GPa}, v=1 / 3$, $a=1.0 \mathrm{~m}$ and $t=0.01 \mathrm{~m}$. The skew angle and the aspect ratio $(a / b)$ of the plate have been separately indicated while presenting the results.

The convergence of the present method with the number of functions used to approximate the displacement fields is carried out for CCCC skew plate with $a / b=1$. The convergence study is carried out for deflections at two different locations namely, (0.5, $0.5)$ and $(0.25,0.25)$. It is to be noted that the coordinates of the points are given in normalized form and $(0.5,0.5)$ represents the point at which maximum deflection occurs. The results of the convergence study in terms of normalized displacement $(w / t)$ are shown in Table 1 for two different skew angles, $30^{\circ}$ and $45^{\circ}$ at $p^{*}=7.94$. The numbers in the parentheses provide a break up for the number of the functions for each plate displacements corresponding to two orthogonal directions. Table 1 clearly shows the convergence of displacements with increase in number of functions. All the results presented in this paper are generated using ( $7 \mathrm{X}$ 7) number of functions for each plate displacements.

Table 1: Convergence of displacements in normalized form $(w / t)$ at two different locations of rhombic CCCC skew plate for two different skew angles at $p^{*}=7.94$

\begin{tabular}{|c|c|c|c|c|c|c|}
\hline \multirow[t]{2}{*}{$\theta$} & \multirow{2}{*}{$\begin{array}{c}\text { Normalized } \\
\text { coordinates } \\
(\xi, \eta)\end{array}$} & \multicolumn{5}{|c|}{ Number of functions in two orthogonal directions } \\
\hline & & $4 \times 4$ & $5 \times 5$ & $6 \times 6$ & $7 \times 7$ & $8 \times 8$ \\
\hline \multirow[t]{2}{*}{$30^{0}$} & $(0.5,0.5)$ & 0.0976 & 0.0974 & 0.0974 & 0.0972 & 0.0972 \\
\hline & $(0.25,0.25)$ & 0.0233 & 0.0232 & 0.0232 & 0.0232 & 0.0231 \\
\hline \multirow[t]{2}{*}{$45^{0}$} & $(0.5,0.5)$ & 0.0478 & 0.0478 & 0.0479 & 0.0478 & 0.0478 \\
\hline & $(0.25,0.25)$ & 0.00824 & 0.00827 & 0.00816 & 0.00814 & 0.00807 \\
\hline
\end{tabular}

\subsection{Validation of the present approach}

In order to validate the present formulation, the results from the present analysis for CCCC boundary condition are contrasted with the results available in the existing literature and also with the results generated by the commercial finite element package ANSYS. The results for $w^{*}$ against $p^{*}$ from present study for aspect ratio $2 / 3$ with two different skew angles have been compared with analytical solution (Chia, 1980) and with the results generated by ANSYS (version 8.0) and shown in Figure 3(a). The results in ANSYS are generated using SHELL 63 element with $80 \times 120$ uniform meshes. The plot shows agreement is better with the results of ANSYS. It also indicates that the amount of deviation in the results of Chia (1980) is more pronounced for $60^{\circ}$ skew angle. The validation of the present approach is further shown in Figure 3(b) with the results of Singh and Elaghabash (2003) for rhombic $(a / b=1)$ skew plates having $30^{\circ}$ and $45^{\circ}$ skew angles. The normalized load-deflection behaviour of simply supported rhombic skew plate is also compared with the results of Singh and Elaghabash (2003) for $45^{\circ}$ skew angle and it is shown in Figure 3(c). In view of Figures 3(a) - 3(c), it may be concluded that the present formulation has the accuracy and stability to analyze large deflection behaviour of skew plates. 


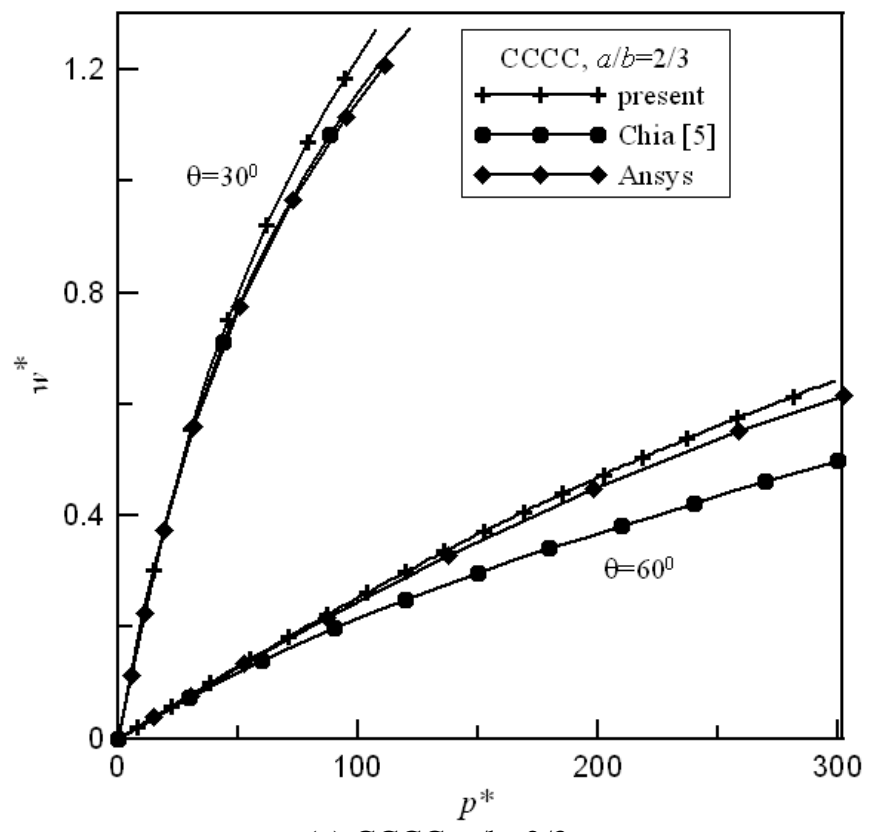

(a) $\mathrm{CCCC}, \mathrm{a} / \mathrm{b}=2 / 3$

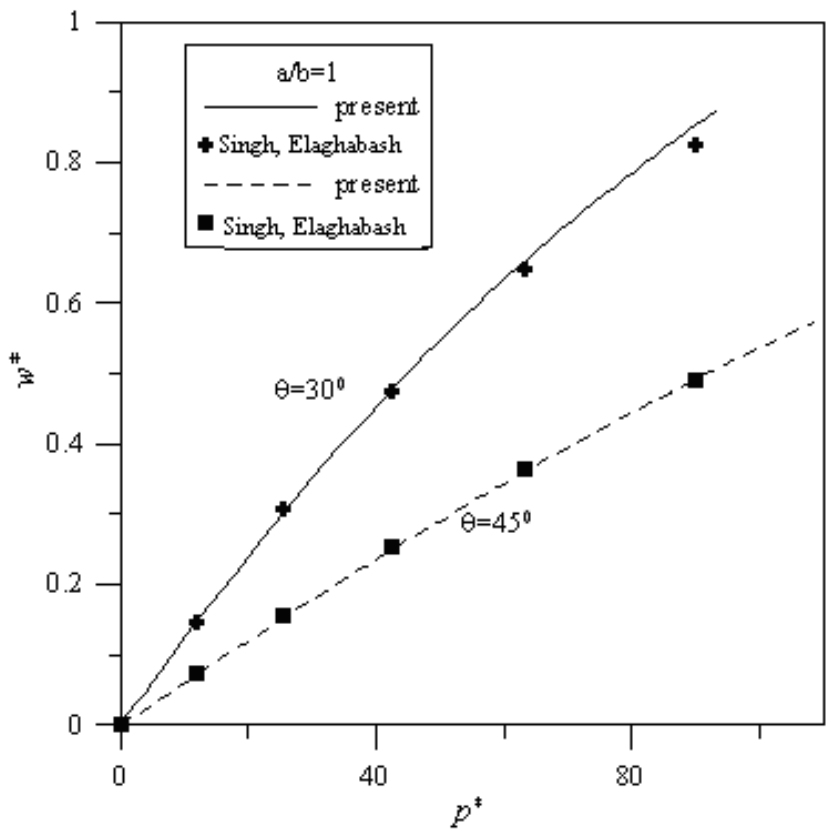

(b) $\mathrm{CCCC}, \mathrm{a} / \mathrm{b}=1$ 


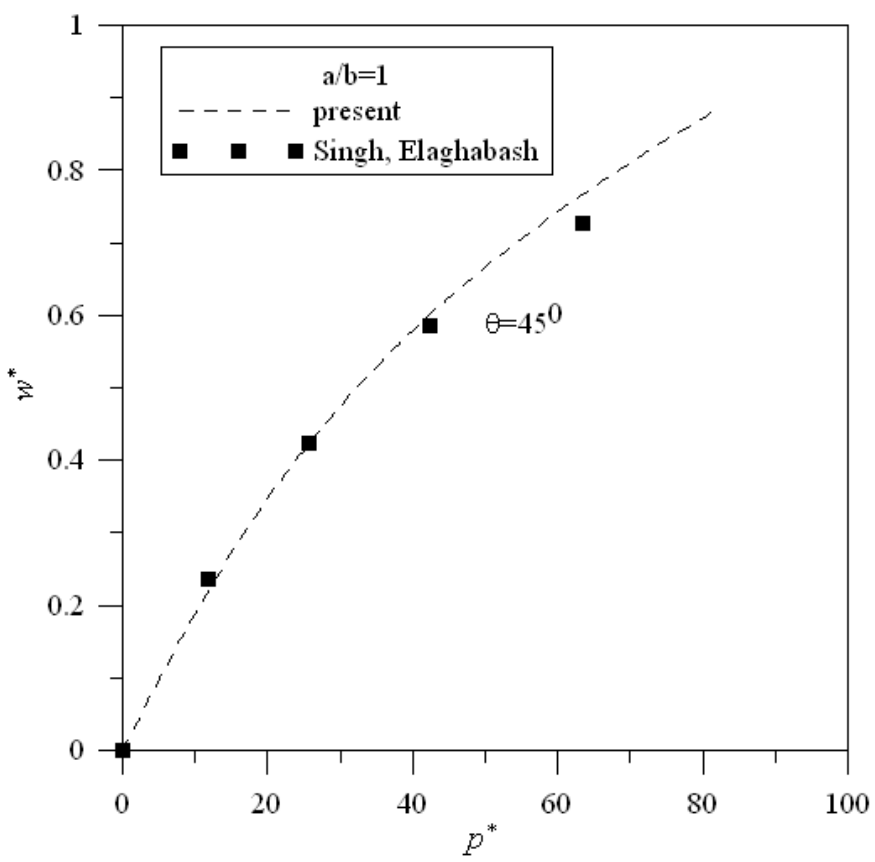

(c) SSSS, $a / b=1$

Figure 3. Validation plots for load-deflection behaviour: (a) CCCC, $a / b=2 / 3$, (b) $C C C C, a / b=1$ and (c) $S S S S, a / b=1$.

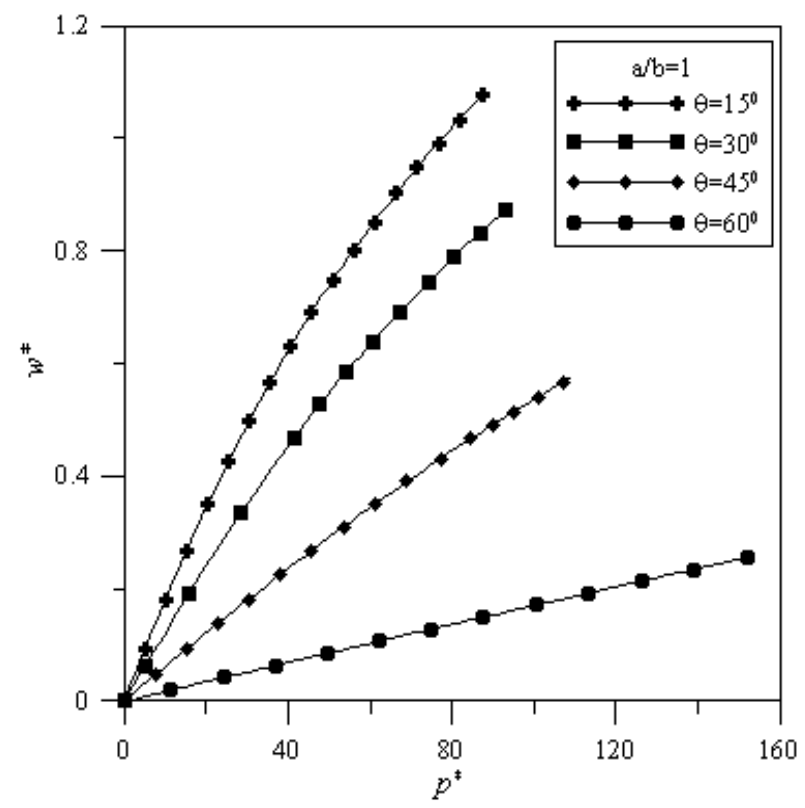

(a) $\mathrm{a} / \mathrm{b}=1$ 


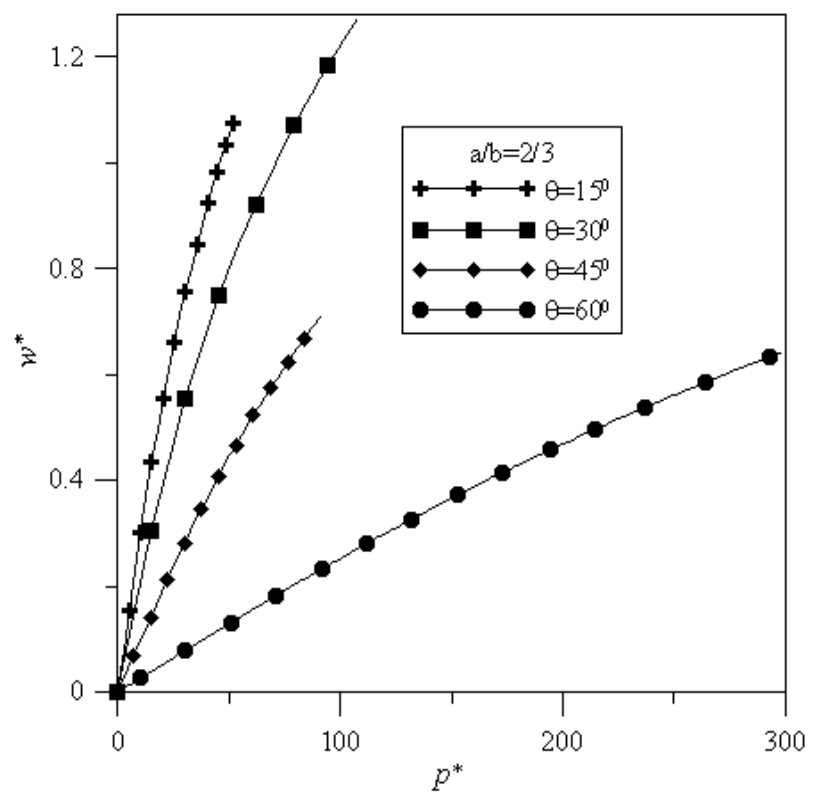

(b) $\mathrm{a} / \mathrm{b}=2 / 3$

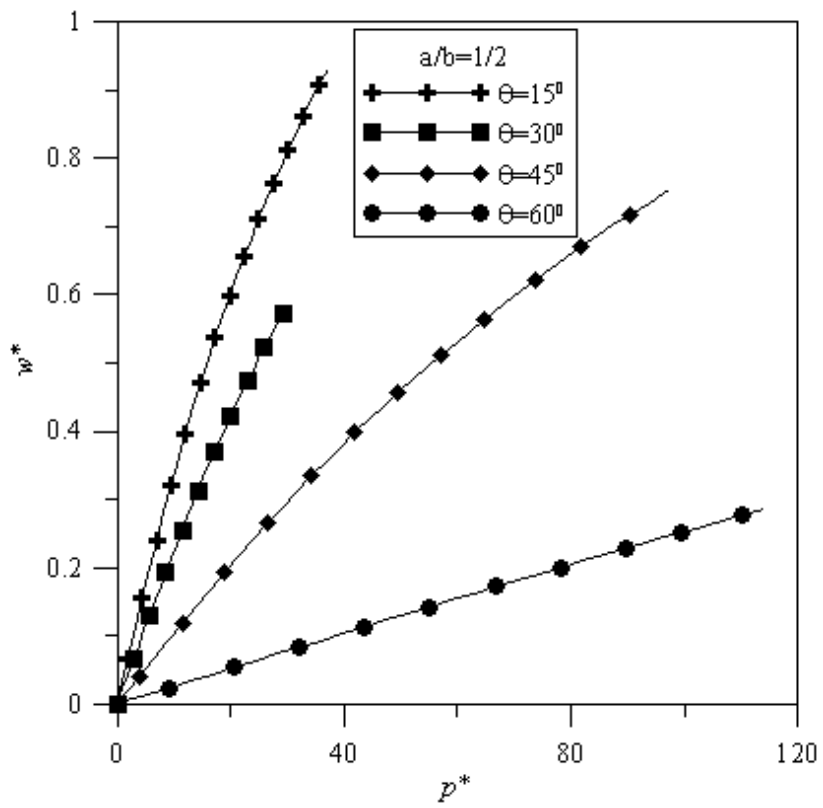

(c) $\mathrm{a} / \mathrm{b}=1 / 2$

Figure 4. Normalized load-deflection plots of CCCC plates with varying aspect ratios and skew angle. 


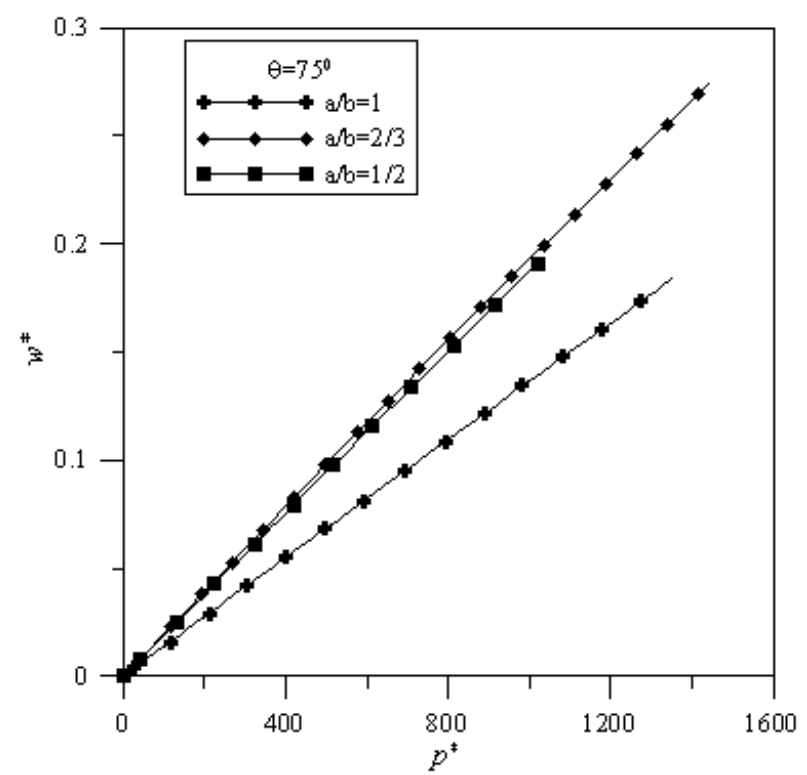

Figure 5. Normalized load-deflection plots of CCCC plates with $75^{0}$ skew angle for varying aspect ratios.

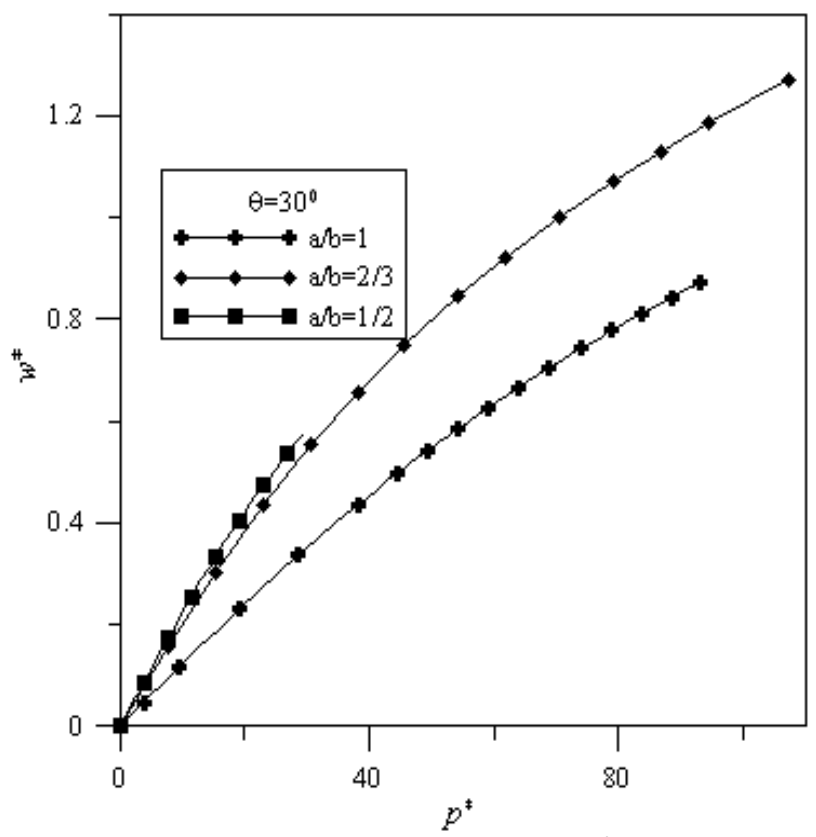

Figure 6. Normalized load-deflection plots of CCCC plates with $30^{\circ}$ skew angle for varying aspect ratios.

\subsection{Results for plate with all edges clamped}

The variation of normalized load-deflection behaviour of skew plates with the variation of skew angles with all edges clamped has been obtained for different values of aspect ratios $(a / b)$. The skew angles selected in each case are $15^{\circ}, 30^{\circ}, 45^{\circ}$ and $60^{\circ}$. Figures 4(a) - 4(c) show $p^{*}$ vs. $w^{*}$ plots for $a / b=1,2 / 3$ and $1 / 2$ respectively. It is to be mentioned that the results for skew angles $15^{\circ}$ and $75^{\circ}$ for all three aspect ratios and for skew angles $30^{\circ}$ and $45^{\circ}$ for aspect ratio $1 / 2$ are new in this paper. It is seen that deflection $\left(w^{*}\right)$ decreases as the skew angle increases for all values of load parameter. This is due to the fact that with increase in skew angles, the system stiffness increases due to increase in stretching force in the middle plane of the plate. Further, for any particular value of load parameter, the slope of the load-deflection plots in the normalized plane decreases with increase in skew angles. This nature of the plots shows a consistent trend for all values of aspect ratio.

The normalized load deflection behaviour for $\theta=75^{0}$ has been separately shown in Figure 5 corresponding to same values of aspect ratios. These plots have not been accommodated in Figure 4 to maintain greater clarity and to avoid significant difference in normalized load scale. However, this plot becomes indicative to show the effect of different aspect ratio for a particular value of 
skew angle in normalized load-deflection behaviour. To get further insight to this phenomenon, another plot is shown in Figure 6 for $30^{\circ}$ skew angle, which also agrees well with the previous trends in results. From the above results, it is quite obvious that the nature of the normalized load-deflection plot tends to a straight line with increase in skew angle and this is true for three different cases of aspect ratio values. Although general trend for normalized load-deflection behaviour for a fixed value of skew angle is shown in Figure 6, it may change significantly as can be seen from the plots for aspect ratio 2/3 and 1/2 in Figures 5 and 6 . A close examination of Figures 5 and 6 reveal that the normalized load-deflection behaviour for aspect ratio 2/3 and 1/2 have interchanged their positions with respect to the same for aspect ratio 1. This appears to be intriguing but similar trend had also been reported in Duan and Mahendran (2003) for $\theta=60^{\circ}$ of clamped skew plate. This finding points towards the existence of a strong interaction among aspect ratio, skew angle and boundary condition on the load-deflection behaviour of the plate in normalized plane, which need further investigation through generation of additional results. It should be mentioned that the normalized loads in Figures 4(ac), Figure 5 and Figure 6 are different because the system stiffness changes with change in skew angle and aspect ratio. Generally, the stiffness of the skew plate increases with increase in skew angle and/ or increase in aspect ratio.

\subsection{Results for plate with all edges simply supported}

The variation of normalized load-deflection behaviour of skew plates with the variation of skew angles with all edges simply supported has been obtained for different fixed values of aspect ratios $(a / b)$ and they are shown in Figs. 7(a) -7 (c) for $a / b=1$, $2 / 3$ and $1 / 2$ respectively. The trends for these plots remain the same as discussed for CCCC plates. It is to be mentioned that the results for skew angles $15^{\circ}$ and $75^{\circ}$ for all three aspect ratios, for skew angles $30^{\circ}$ and $45^{\circ}$ for aspect ratio $2 / 3$ and for skew angles $45^{0}$ for aspect ratio $1 / 2$ are new in this paper.

The normalized load deflection behaviour with the variation of aspect ratio for $\theta=75^{0}$ has also been shown separately in Figure 8 due to the same reason mentioned in section 4.2. A similar plot for $\theta=45^{0}$ has been shown in Figure 9. Both these plots show that for a particular value of skew angle, the slope of the normalized load-deflection plot increases with decrease in aspect ratio. Unlike the case of clamped plates for $\theta=75^{\circ}$ (Figure 5), the normalized load-deflection behaviour for simply supported plates with similar skew angle show a consistent trend in conformity with the general behaviour as mentioned in section 4.2

In order to visualize the static deflection of the plate, an enlarged plot for deflected position of a $60^{\circ}$ skew plate with clamped boundary for aspect ratio 2/3 has been shown in Figure 10. It is to be noted that the deflected shape of the plate is represented by considering only the vertical displacement. Singh and Elaghabash (2003) indicated that there are many numerical results in the literature but only a limited quantity of them is truly available. Moreover it is found during computation that the convergence of the solution is really a critical issue. In general, it is found that the convergence of the solution has serious difficulties for skew angles $60^{\circ}$ and above, and in such situations an assumed displacement field extrapolated from previous solutions, yields better convergence. It should be noted that the normalized loads in Figures 7(a-c), Figure 8 and Figure 9 are different due to the same reason stated in section 4.2 for clamped plate.

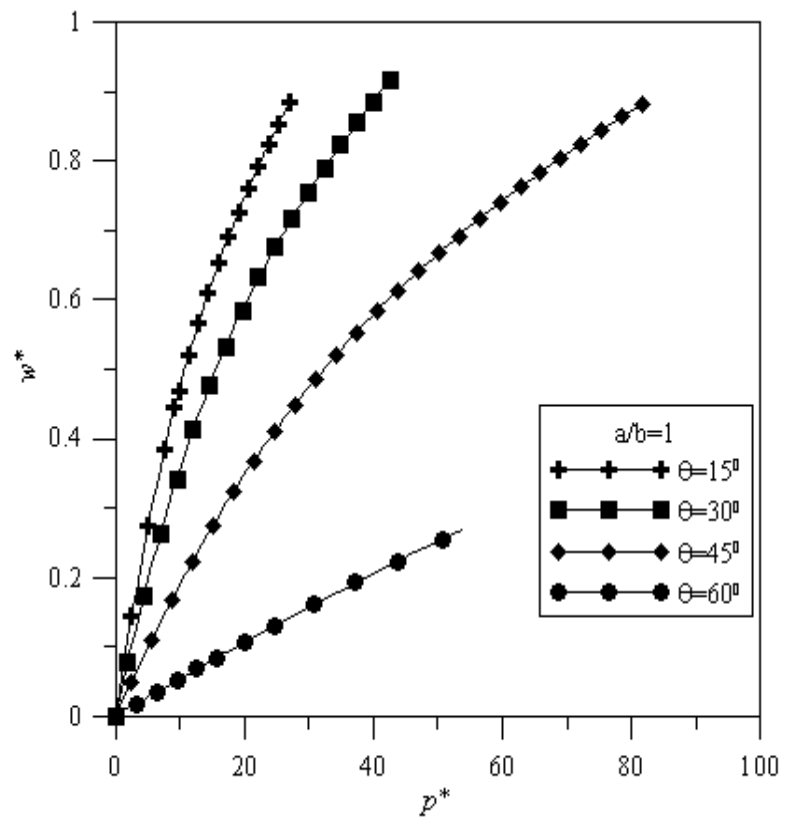

(a) $a / b=1$ 


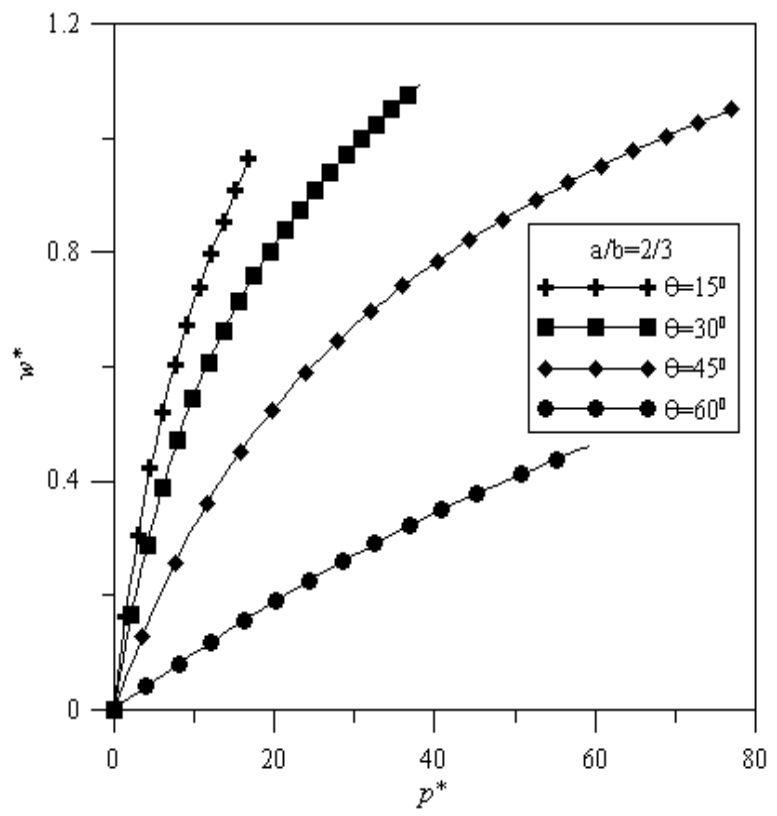

(b) $\mathrm{a} / \mathrm{b}=2 / 3$

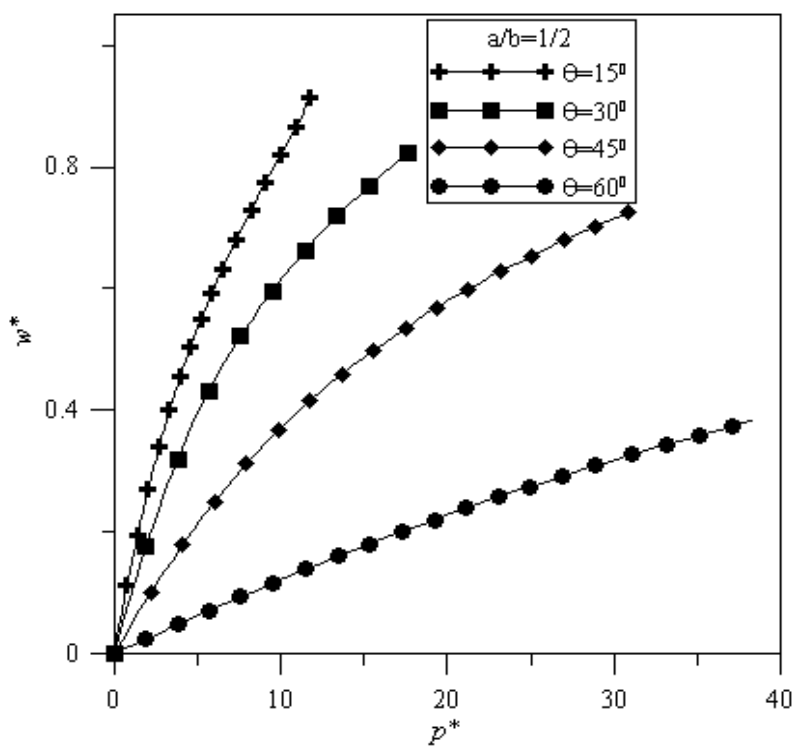

(c) $\mathrm{a} / \mathrm{b}=1 / 2$

Figure 7. Normalized load-deflection plots of SSSS plates with varying aspect ratios and skew angle. 


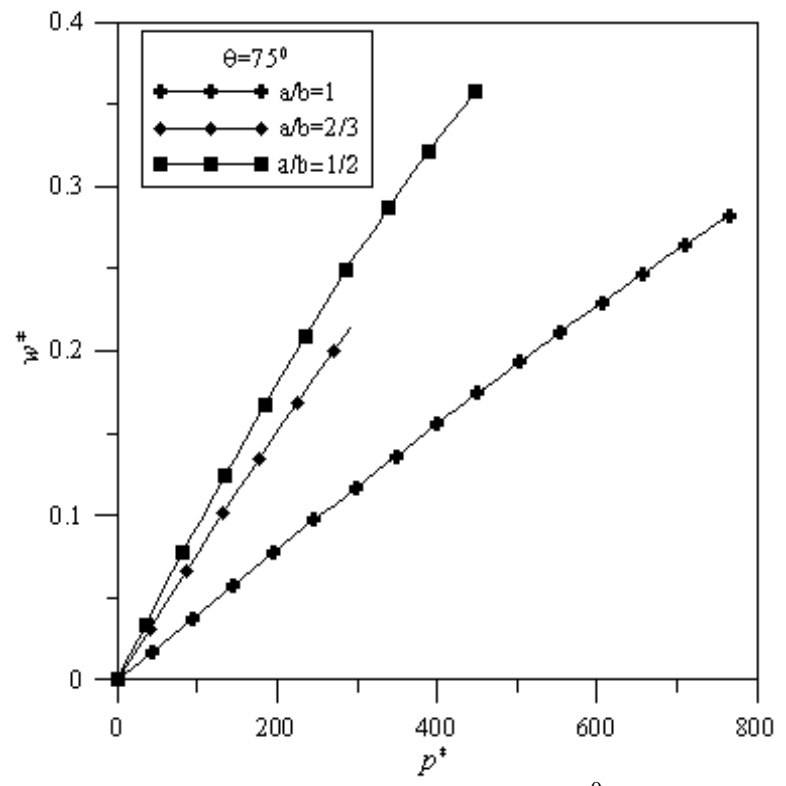

Figure 8. Normalized load-deflection plots of SSSS plates with $75^{\circ}$ skew angle for varying aspect ratios.

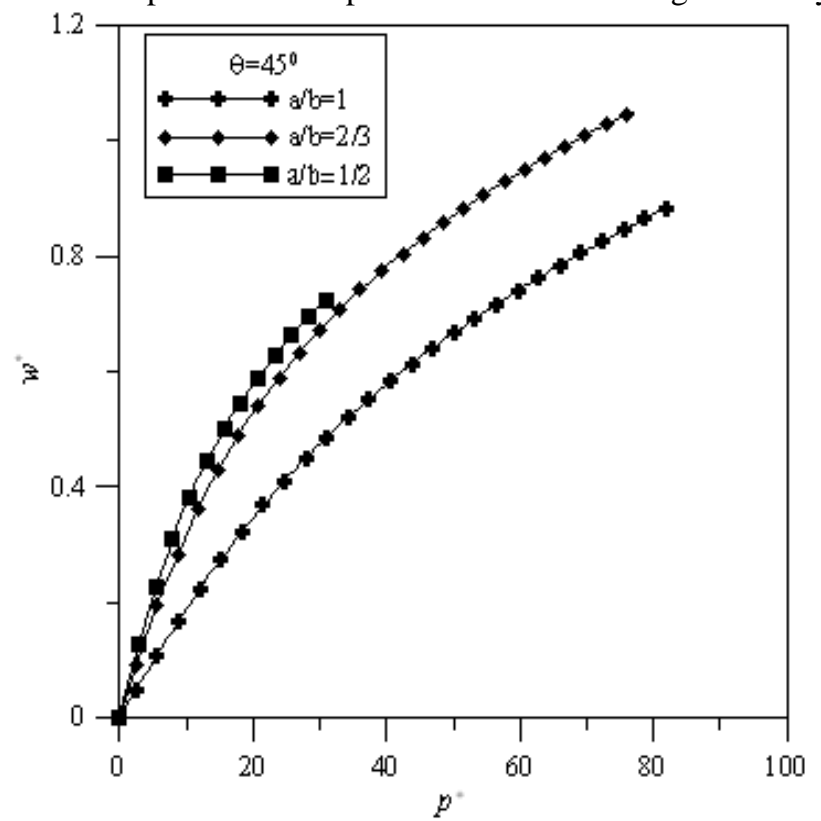

Figure 9. Normalized load-deflection plots of SSSS plates with $45^{\circ}$ skew angle for varying aspect ratios.

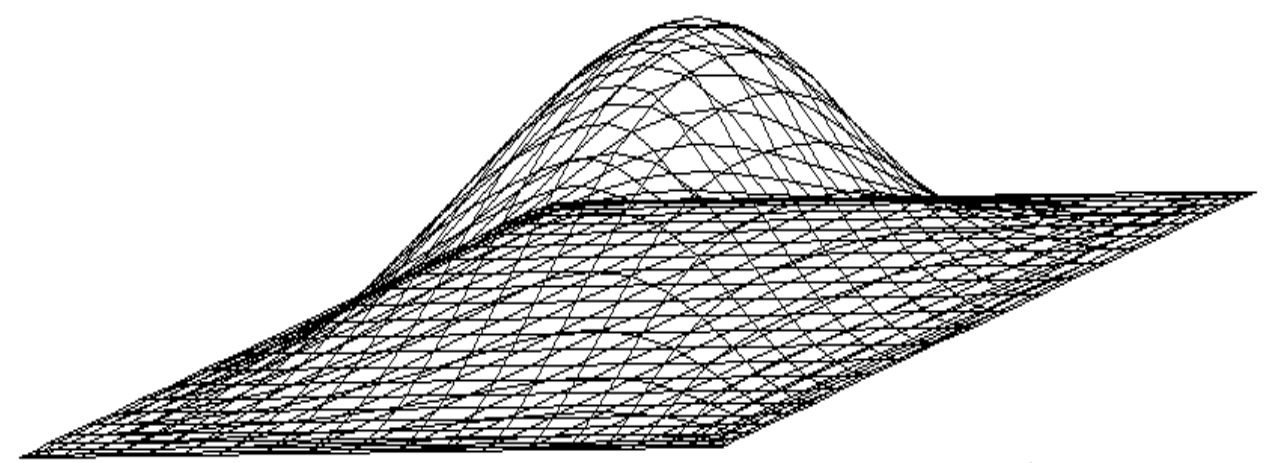

Figure 10. Enlarged view of deflected shape of a CCCC plate with $60^{\circ}$ skew angle. 


\section{Conclusions}

In this paper, a simulation model for large deflection static analysis of a thin isotropic skew plate under uniform transverse pressure has been presented based on variational form of energy principle. The displacement fields are assumed as admissible orthogonal polynomials and the resulting nonlinear set of equations is solved by direct substitution method using relaxation parameter. The validation of the present method is carried out successfully with the available results, thus establishing the accuracy of the present method. The results indicate a strong interaction between aspect ratio and skew angle on the load-deflection behaviour of skew plates. Also, a new extrapolation technique for the solution of the set of nonlinear algebraic equations has been introduced. A good amount of new results are presented in this paper. This method of analysis establishes the basic kernel, which can be extended to various other types of static and dynamic skew plate problems.

\section{Nomenclature}

a Length of the plate

$b \quad$ Width of plate along skew direction

$\{d\} \quad$ Vector of unknown coefficients

$D \quad$ Flexural rigidity of the plate

$E \quad$ Young's modulus of the plate

$\{f\} \quad$ Load vector

$J \quad$ Jacobian of the coordinate transformation

$[K] \quad$ Stiffness matrix

$\left[k^{b}\right] \quad$ Stiffness matrix due to bending

$\left[k^{m}\right] \quad$ Stiffness matrix due to stretching

$n w \quad$ Number of functions for $w$

$n u \quad$ Number of functions for $u$

$n v \quad$ Number of functions for $v$

$p$ Uniform transverse pressure

$p^{*} \quad$ Non-dimensional load parameter

$t \quad$ Thickness of the plate

$u \quad$ Displacement along $x$ direction

$U \quad$ Strain energy

$U_{b} \quad$ Strain energy due to flexural action

$U_{m} \quad$ Strain energy due to membrane action

$v \quad$ Displacement along $y$ direction

$V \quad$ Work potential

w Transverse displacement

$w^{*} \quad$ Maximum transverse displacement

$v \quad$ Poisson's ratio

$\phi_{i} \quad$ Set of functions defining approximate displacement field $w$

$\alpha_{i} \quad$ Set of functions defining approximate displacement field $u$

$\beta_{i} \quad$ Set of functions defining approximate displacement field $v$

$\theta \quad$ Skew angle

\section{References}

Alwar R.S. and Rao N.R. 1974. Large elastic deformations of clamped skewed plates by dynamic relaxation. Computers \& Structures, vol. 4, pp.381-398.

Alwar R.S. and Rao N.R. 1973. Nonlinear analysis of orthotropic skew plates. AIAA Journal, vol.11, No.4, pp. 495-498.

Ashour A.S. 2009. The free vibration of symmetrically angle-ply laminated fully clamped skew plates, Journal of Sound and Vibration, vol. 323, No. 1-2, pp. 444-450.

Buragohain D.N. and Patodi S.C. 1978. Large deflection analysis of skew plates by lumped triangular element formulation. Computers \& Structures, vol.9, No.2, pp.183-189.

Chia C. (1980): Nonlinear analysis of plates. USA:McGraw-Hill Inc.

Cook R.D., Malkus D.S. and Plesha M.E. 1989. Concepts and applications of finite element analysis. USA: John Wiley and Sons.

Daripa R., Singha, M.K. 2009. Influence of corner stresses on the stability characteristics of composite skew plates, International Journal of Non-Linear Mechanics, vol. 44, No. 2, pp.138-146. 
Das D., Sahoo P., Saha K. 2008. Large-amplitude dynamic analysis of simply supported skew plates by a variational method, Journal of Sound and Vibration, vol. 313, No. 1-2, pp.246-267.

Das D., Sahoo P., Saha K. 2009. Nonlinear vibration analysis of clamped skew plates by a variational method, Journal of Vibration Control, vol. 15, pp.985-1017.

Duan M. and Mahendran M. 2003. Large deflection analyses of skew plates using hybrid/mixed finite element method.

Computers \& Structures, vol.81, pp.1415-1424.

Liew K.M. and Han J.B. 1997. Bending analysis of simply supported shear deformable skew plates. Journal of Engineering Mechanics, vol.123, No.3, pp.214-221.

Liew K.M., Chen X.L. and Reddy J.N. 2004. Mesh-free radial basis function method for buckling analysis of non-uniformly loaded arbitrarily shaped shear deformable plates. Computers Methods in Applied Mechanics and Engineering, vol.193, pp.205224.

Malekzadeh P. 2007. A differential quadrature nonlinear free vibration analysis of laminated composite skew thin plates, ThinWalled Structures, vol. 45, No. 2, pp.237-250.

Malekzadeh P. 2008. Differential quadrature large amplitude free vibration analysis of laminated skew plates based on FSDT, Composite Structures, vol. 83, No. 2, pp.189-200.

Malekzadeh P. and Foiuz A.R. 2007. Large deformation analysis of orthotropic skew plates with nonlinear rotationally restrained edges using DQM. Composite Structures, vol.80, No.2, pp.196-206.

Malekzadeh P., Karami G. 2006. Differential quadrature nonlinear analysis of skew composite plates based on FSDT, Engineering Structures, vol. 28, No. 9, pp.1307-1318.

Muhammad T., Singh A. V. 2004. A p-type solution for the bending of rectangular, circular, elliptic and skew plates, International Journal of Solids and Structures, vol. 41, No. 15, pp. 3977-3997.

Prakash T., Singha M.K., Ganapathi M. 2008. Thermal postbuckling analysis of FGM skew plates, Engineering Structures, vol. 30, No. 1, pp.22-32.

Rajamohan C. and Raamachandran J. 1997. Boundary element analysis of skew plates using a new fundamental solution. Mechanics Research Communications, vol.24, No.4, pp.407-414.

Saadatpour M.M., Azhari M. and Bradford M.A. 1998. Buckling of arbitrary quadrilateral plates with intermediate supports using the Galerkin method. Computer Methods in Applied Mechanics and Engineering, vol.164, pp.297-306.

Singh A.V. and Elaghabash Y. 2003. On finite displacement analysis of quadrangular plates. International Journal of Non Linear Mechanics, vol.38, pp.1149-1162.

Singha M. K., Ramachandra L.S., Bandyopadhyay J.N. 2006. Vibration behavior of thermally stressed composite skew plate, Journal of Sound and Vibration, vol. 296, No. 4-5, pp.1093-1102.

Srinivasan R.S. and Ramachandran S.V. 1976. Large deflection of clamped skew plates. Computer Methods in Applied Mechanics and Engineering, vol.7, No.2, pp.219-233.

Timoshenko S.P. and Woinowsky K.S. 1959. Theory of plates and shells. Singapore: McGraw Hill International Editions.

Wang X., Tan M. and Zhou Y. 2003. Buckling analyses of anisotropic plates and isotropic skew plates by the new version differential quadrature method.Thin-Walled Structures, vol.41, pp.15-19.

Wu G.Y., Shih Y.S. 2006. Analysis of dynamic instability for arbitrarily laminated skew plates, Journal of Sound and Vibration, vol. 292, No. 1-2, pp.315-340.

Xiang Y., Wang C.M. and Kitipornchai S. 1995. Buckling of skew Mindlin plates subjected to in-plane shear loadings. International Journal of Mechanical Sciences, vol.37, No.10, pp.1089-1101.

\section{Biographical notes}

Debabrata Das graduated from the Department of Mechanical Engineering, R. E. C., Durgapur and got his Master in Mechanical Engineering degree from Jadavpur University. He has served the national Defence Research and Development Organization for five years as a scientist. Thereafter he started pursuing research in the area of large amplitude free and forced vibration analysis of various structural elements in elastic and post-elastic regime. He has completed his $\mathrm{Ph}$. D. thesis from Jadavpur University. He has 11 publications in international journals and conference proceedings. At present he serves as Senior Lecturer in M. S. Institute of Technology, Kolkata, India.

Prasanta Sahoo is a Professor at the Department of Mechanical Engineering, Jadavpur University, Kolkata, India. He received Bachelor of Mechanical Engineering from Jadavpur University and MTech and PhD in Mechanical Engineering from Indian Institute of Technology, Kharagpur, India. His research area is tribology in manufacturing, contact mechanics and structural mechanics. He is the recipient of First Track Project for Young Scientists from Department of Science and Technology, India. He is the supervisor of 5 PhD theses and 15 Master's theses. He has authored a textbook on Engineering Tribology (Prentice Hall of India, 2005) and a book-chapter in Tribology Research Advances (Nova Science, 2009). He has co-authored more than 120 technical papers in international journals and conference proceedings. He is on the editorial board of Journal of Tribology Research (Serials), Journal of Tribology and Surface Engineering (Nova Science), Materials and Design (Elsevier).

Kashi Nath Saha graduated from the Department of Mechanical Engineering, Jadavpur University and then served Indian industry for five years as design engineer. Joined academia as lecturer in the Department of Mechanical Engineering, Jadavpur University in 1987 and pursuing teaching and research in the area of Machine Elements Design and Computer aided drafting, Stress, deformation analysis and design of Structural 
Elements and Structural Stability Analysis. He has also carried out several experimental studies in the field of structural dynamics and supervised a number of post graduate thesis and research projects and has over 50 publications in international journals and conference proceedings. At present he serves as Associate Professor in the Department of Mechanical Engineering, Jadavpur University.

Received May 2009

Accepted July 2009

Final acceptance in revised form August 2009 